\title{
Inference of topology and the nature of synapses, and the flow of information in neuronal networks
}

\author{
F. S. Borges, ${ }^{1,2}$ E. L. Lameu, ${ }^{3}$ K. C. Iarosz,${ }^{1,4}$ P. R. Protachevicz,${ }^{5}$ I. L. Caldas, ${ }^{1}$ R. L. Viana, ${ }^{6}$ E. E. N. Macau,, 37 \\ A. M. Batista, ${ }^{1,4,5,8}$ and M. S. Baptista ${ }^{4}$ \\ ${ }^{1}$ Physics Institute, University of São Paulo, São Paulo, SP 05508-090, Brazil \\ ${ }^{2}$ Center of Mathematics, Computation and Cognition, Federal University of ABC, São Bernardo do Campo, SP 09606-045, Brazil \\ ${ }^{3}$ National Institute for Space Research, São José dos Campos, SP 12227-010, Brazil \\ ${ }^{4}$ Institute for Complex Systems and Mathematical Biology, University of Aberdeen, SUPA, AB24 3FX, United Kingdom \\ ${ }^{5}$ Post-Graduation in Science, State University of Ponta Grossa, Ponta Grossa, PR 84030-900, Brazil \\ ${ }^{6}$ Physics Department, Federal University of Paraná, Curitiba, PR 81531-980, Brazil \\ ${ }^{7}$ Federal University of São Paulo, São José dos Campos, SP 12231-280, Brazil \\ ${ }^{8}$ Mathematics and Statistics Department, State University of Ponta Grossa, Ponta Grossa, PR 84030-900, Brazil
}

(Received 28 September 2017; revised manuscript received 21 December 2017; published 7 February 2018)

\begin{abstract}
The characterization of neuronal connectivity is one of the most important matters in neuroscience. In this work, we show that a recently proposed informational quantity, the causal mutual information, employed with an appropriate methodology, can be used not only to correctly infer the direction of the underlying physical synapses, but also to identify their excitatory or inhibitory nature, considering easy to handle and measure bivariate time series. The success of our approach relies on a surprising property found in neuronal networks by which nonadjacent neurons do "understand" each other (positive mutual information), however, this exchange of information is not capable of causing effect (zero transfer entropy). Remarkably, inhibitory connections, responsible for enhancing synchronization, transfer more information than excitatory connections, known to enhance entropy in the network. We also demonstrate that our methodology can be used to correctly infer directionality of synapses even in the presence of dynamic and observational Gaussian noise, and is also successful in providing the effective directionality of intermodular connectivity, when only mean fields can be measured.
\end{abstract}

DOI: 10.1103/PhysRevE.97.022303

\section{INTRODUCTION}

Many real systems have been modeled by complex networks with different topological characteristics. Network theory has been applied in a large number of examples and different research fields, such as biology [1], economics [2], and physics [3]. In neuroscience, the application of network theory provides a way to analyze the structure and the functional behavior of neuronal systems [4]. A fundamental research topic in neuroscience is the determination of the brain structure, to better understand its functioning. Some neuronal networks had their structure directly mapped by means of diffusion tensor imaging tractography [5].

One of the most challenging problems in neuronal networks is the inference of its topology, that is, the determination of the underlying synaptic connectivity by indirect means, based on functional measurements of time series of the membrane potential $[6,7]$. There are works that infer the topology based on functional measures such as correlation [8,9] and synchronization [10], or functional magnetic resonance imaging [11]. And there are those based on informational quantities [12-14,17,18]. Inference based on functional measures requires a threshold analysis that establishes a link between the measurement and the physical connection $[12,19,20]$. Rubido et al. [17] showed that a threshold can be calculated whenever a functional measure between nodes [cross correlation (CC) or mutual information] in a network is dissimilar. Higher functional values correspond to a pair of adjacent nodes, lower functional values to nonadjacent nodes. Bianco-Martinez et al. [18] used the mutual information rate (MIR) to successfully infer the connectivity of a network composed of HindmarshRose (HR) neurons [21] connected by electrical synapses. Both works in Refs. $[17,18]$ have shown that the threshold technique could surprisingly provide an inferred network that matched exactly with the real network. These works have considered undirected networks, where nodes were connected bidirectionally with the same intensity.

This work considers HR networks with chemical synapses. Unlike electrical synapses that are undirected, chemical synapses are directed [22]. Whereas undirected networks can have their topologies properly inferred by CC and MIR, directed networks require methodologies capable of detecting the directionality of the physical influence $[12,19,23]$. Granger causality [12] is a concept construct on the idea that one can obtain optimal fittings of mathematical models about the measured time series that provide the structure and direction of the connectivity. These models are statistically optimized to improve the predictability of events in one time series based on observations of other time series and have been shown to be a powerful tool to infer [24]. Informational quantities have also been demonstrated to provide a framework that is at the frontier to infer. In Ref. [17] it was shown that inference based on mutual information is more reliable than those based on correlational measurements. In Ref. [23] it was shown that directed information had advantages over Granger causality for quantifying effective connectivity in the brain. 
One question that remains open is whether information measures can reliably infer the connectivity of complex neuronal networks for all existing synapses by only accessing bivariate measurements, in contrast to more complex and computational demanding techniques such as multivariate analysis based on informational analysis [14,15], a technique that takes into consideration time series from more than two neurons at each time, or modeled-based multivariate approaches such as those that employ compressive sensing [16].

In this work, we use the recently defined causal mutual information (CaMI) [25,26] calculated using an appropriate methodology to infer the direction of chemical synapses in complex neuronal networks without any mistake, by only considering easy to handle and to measure bivariate time series. Moreover, we show that inhibitory connections are responsible for a considerably larger amount of information transfer than that compared to neurons connected by excitatory synapses. This allows one to infer also the nature of the connection (excitatory and inhibitory), and not only its existence as previous techniques. The role of the inhibitory connections have been also found by Montani et al. [27] to have a critical role on the information transmission. That is, when a population of neurons is considered with an important number of inhibitory neurons, they help to rapidly phase lock neural populations and induce synchronization at small time windows and produce stable firing patterns. Inhibitory neurons can easily help to regulate the level of activity of other neurons. Furthermore, we will also show that nonadjacent neurons transmit roughly null amount of directed information, indicating that indeed causal information has a direct relationship with the existence of a synapse.

The CaMI was constructed from the idea that if there is a flow of information from system A to system B, then longer time series (or measurements with higher precision) from $\mathrm{B}$ should have a positive mutual information to short time series (or to observations with lower precision) in A. This quantity, measuring the influence from $\mathrm{A}$ to $\mathrm{B}$, was shown to be equal to the transfer entropy (TE) [13] from A to B plus the mutual information between $\mathrm{A}$ and $\mathrm{B}$ when both systems are being measured with the same resolution. The advantage of CaMI, however, is that it allows one to calculate TE, and therefore the directionality of the flow of information, by using measurements with arbitrary resolution, which in turn also allows for the correct calculation of the TE using binary partitions of the phase space, i.e., appropriated when measurements have the lowest possible resolution. Moreover, CaMI can be calculated in lower-dimensional space of only two dimensions, without the need to consider conditional probabilities, but only marginal and joint probabilities, and finally, it is a quantity that fully expresses not only the exchange of information (MIR), but also its causal directionality (TE).

\section{NEURONAL NETWORK}

\section{A. Hindmarsh-Rose network}

We consider the random neuronal network (RNN) [28,29] introduced by Gelenbe [30] and the neuronal network of the nematode worm $C$. elegans [31] whose structure was completely mapped at a cellular level [32]. The node dynamics in the network is expressed by the Hidmarsh-Rose (HR) neuron model. Hindmarsh and Rose [21] proposed a phenomenological neuron model that is a simplification of the HodgkinHuxley model [33]. The HR is described by

$$
\begin{aligned}
& \dot{p}=q-a p^{3}+b p^{2}-n+I_{\mathrm{ext}}, \\
& \dot{q}=c-d p^{2}-q, \\
& \dot{n}=r\left[s\left(p-p_{0}\right)-n\right],
\end{aligned}
$$

where $p(t)$ is the action potential of the membrane, $q(t)$ is related to the fast current, $\mathrm{K}^{+}$or $\mathrm{Na}^{+}$, and $n(t)$ is associated with the slow current, for instance, $\mathrm{Ca}^{2+}$. We use the parameters $a=1, b=3, c=1, d=5, s=4, r=0.005$, $p_{0}=-1.60$, and $3.24 \leqslant I_{\text {ext }} \leqslant 3.25$, so that the HR neuron exhibits a chaotic burst behavior. Presynaptic neurons with an action potential $p_{j}$ coupled by chemical synapses to neurons $i$ modifying its action potential $p_{i}$ according to

$$
\begin{aligned}
\dot{p}_{i}= & q_{i}-a p_{i}^{3}+b p_{i}^{2}-n_{i}+I_{\mathrm{ext}} \\
& +g_{c}\left(V_{\mathrm{syn}}-p_{i}\right) \sum_{j=1}^{N} \varepsilon_{i j} \Gamma\left(p_{j}\right),
\end{aligned}
$$

where $(i, j)=1, \ldots, N, N$ is the neurons number, and $g_{c}$ is the chemical coupling strength. The chemical synapse function is modeled by the sigmoidal function,

$$
\Gamma\left(p_{j}\right)=\frac{1}{1+\exp \left[-\lambda\left(p_{j}-\Theta_{\mathrm{syn}}\right)\right]},
$$

with $\Theta_{\text {syn }}=1.0, \lambda=10, V_{\text {syn }}=2.0$ for excitatory and $V_{\text {syn }}=$ -1.5 for inhibitory synapses. The adjacency matrix $\varepsilon_{i j}$ describes the neurons chemically connected.

\section{B. Encoding the trajectory into symbolic sequences}

To do our analysis, we normalize $p_{i}$ through the equation,

$$
x_{i}=\frac{p_{i}^{\max }-p_{i}}{p_{i}^{\max }-p_{i}^{\min }},
$$

where $p_{i}^{\max }$ and $p_{i}^{\min }$ are the maximum and minimum values, respectively, of the time series of $p_{i}(t)$. Figure 1 shows the normalized membrane potential for two chemical coupled HR neurons with connection from $x_{2}$ (red squares) to $x_{1}$ (black circles). The black and red circles correspond to $X_{1}$ and $X_{2}$, respectively, where the mapping step time $\Delta t$ is equal to $1 \mathrm{~ms}$. With the forward-time trajectory $X_{i}^{L}(n)=$ $x_{i}^{n}, \ldots, x_{i}^{n+L-1}$, where $L$ is the length of the time series $X(n)$ and $n$ is the discrete time, we generate a symbolic sequence $S_{i}^{L}(n)=s_{i}^{n}, \ldots, s_{i}^{n+L-1}$, where we consider $s_{i}^{n}=0$ if $x_{i}^{n} \leqslant 0.5$ and $s_{i}^{n}=1$ if $x_{i}^{n}>0$.

Table I exhibits some mapped values of $x_{1}^{n}$ and $x_{2}^{n}$ with their respective length-1 symbolic values $s_{1}^{n}$ and $s_{2}^{n}$, and also the length-2 and length-4 symbolic sequence $S_{1}^{L=2}(n)$ and $S_{2}^{L=4}(n)$, respectively.

Considering the symbolic sequences $S_{i}^{L}$ and $S_{j}^{2 L}$ is possible to find the probabilities $P\left(S_{i}^{L}\right), P\left(S_{j}^{2 L}\right)$, and $P\left(S_{i}^{L}, S_{j}^{2 L}\right)$. These probabilities are used to calculate the casual mutual information. 


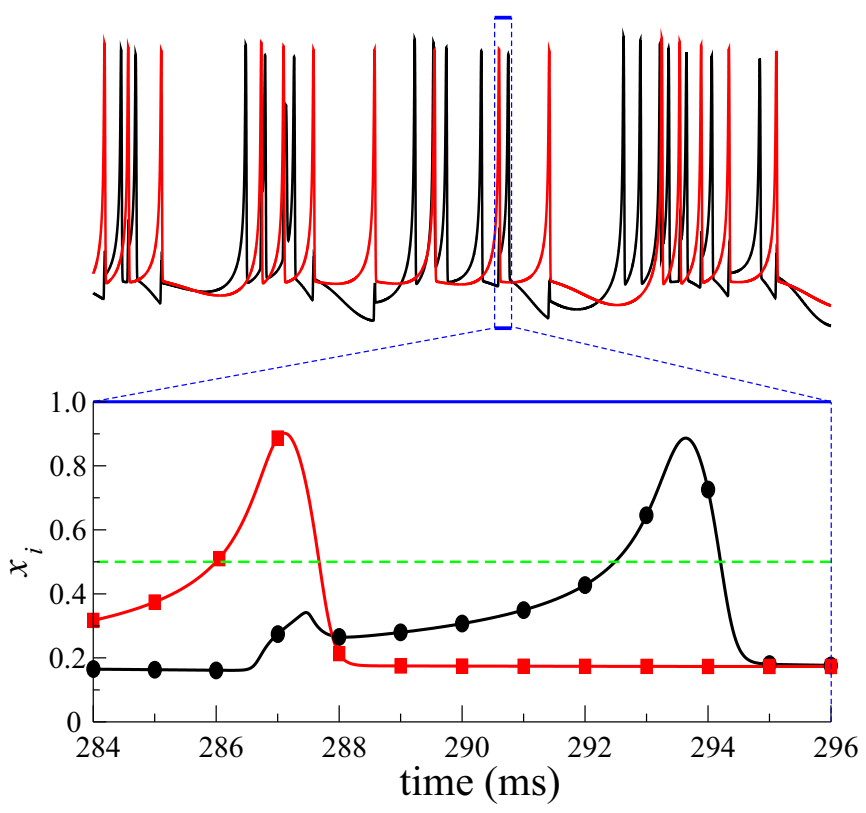

FIG. 1. Normalized membrane potential of two chemical coupled HR neurons with connection from $x_{2}$ (red line) to $x_{1}$ (black line). We consider the coupling strength $g_{c}=1$ and the mapping time step $\Delta t=1 \mathrm{~ms}$. The black circles and red squares correspond to $X_{1}$ and $X_{2}$, respectively.

\section{CASUAL MUTUAL INFORMATION}

In order to be able to describe most of the information content of the time series by a short-length binary symbolic representation, we make a time-Poincaré map of the time series. Ideally, in the case one wants short symbolic sequences to fully express the amount of information of infinitely long sequences, points in the mapping should be spaced by a time step such that the symbolic representation of the time series behaves as a random process, i.e., the next symbolic sequence is decorrelated with the previous. We are interested in obtaining a good estimation of CaMI to correctly infer the network's topology, its synaptic nature, and to obtain a sufficiently accurate value for the magnitude of the flow of information (e.g., CaMI, MIR, and TE). Given a time step $\Delta t$, a mapping for neuron $i X_{i}$ is constructed by collecting a point of the membrane potential at times $t=n \Delta t$ producing the discrete

TABLE I. Mappings for $\Delta t=1 \mathrm{~ms}$ and $L=2$.

\begin{tabular}{lcccccc}
\hline \hline $\mathbf{n}$ & $\boldsymbol{x}_{\mathbf{1}}^{\boldsymbol{n}}$ & $\boldsymbol{s}_{\mathbf{1}}^{\boldsymbol{n}}$ & $\boldsymbol{S}_{\mathbf{1}}^{L=\mathbf{2}}(\boldsymbol{n})$ & $\boldsymbol{x}_{\mathbf{2}}^{\boldsymbol{n}}$ & $\boldsymbol{s}_{\mathbf{2}}^{\boldsymbol{n}}$ & $\boldsymbol{S}_{\mathbf{2}}^{L=\mathbf{4}}(\boldsymbol{n})$ \\
\hline 285 & 0.163043 & 0 & 00 & 0.374431 & 0 & 0110 \\
286 & 0.161350 & 0 & 00 & 0.500448 & 1 & 1100 \\
287 & 0.274266 & 0 & 00 & 0.886694 & 1 & 1000 \\
288 & 0.265589 & 0 & 00 & 0.213396 & 0 & 0000 \\
289 & 0.279589 & 0 & 00 & 0.174788 & 0 & 0000 \\
290 & 0.306991 & 0 & 00 & 0.174349 & 0 & 0000 \\
291 & 0.349396 & 0 & 00 & 0.173966 & 0 & 0000 \\
292 & 0.427650 & 0 & 01 & 0.173642 & 0 & 0000 \\
293 & 0.645130 & 1 & 11 & 0.173384 & 0 & - \\
294 & 0.725724 & 1 & 10 & 0.173200 & 0 & - \\
295 & 0.180110 & 0 & - & 0.173100 & 0 & - \\
\hline \hline
\end{tabular}

time series described $x_{i}^{n}=x_{i}(t=n \Delta t)$. In this way, we obtain the mapping $X_{i}=x_{i}^{0}, x_{i}^{1}, x_{i}^{2}, \ldots, x_{i}^{T-1}$ for neuron $i$, where $T$ is the number of points in the mapping. In the following, we will study coupled neurons to determine a time step for which CaMI is maximized, aiming with this maximization to construct a time-Poincaré map that tends to behave as a Markov process, allowing CaMI, MI, and TE to express a good approximate of their real values.

Bianco-Martinez and Baptista [25,26] defined a new quantity named CaMI from $X_{i}$ to $X_{j}\left(C_{X_{i} \rightarrow X_{j}}\right)$ as the MI $(I)$ between joint events in $X_{i}^{-L}$ and the set composed by the joint events of $X_{j}^{-L}$ and $X_{j}^{L}$ as

$$
C_{X_{i} \rightarrow X_{j}}=I\left(X_{i}^{-L} ;\left(X_{j}^{-L}, X_{j}^{L}\right)\right)=I\left(X_{i}^{L} ; W_{j}^{2 L}\right),
$$

where the mutual information $I\left(X_{i}^{L} ; X_{j}^{L}\right)$ is given by

$$
I\left(X_{i}^{L} ; X_{j}^{L}\right)=H\left(X_{i}^{L}\right)+H\left(X_{j}^{L}\right)-H\left(X_{i}^{L}, X_{j}^{L}\right),
$$

and $H\left(X_{i}^{L}\right)$ is the Shannon entropy of length- $L$ trajectory points of the discrete mapping. It is also true that

$$
\mathrm{C}_{X_{i} \rightarrow X_{j}}=I\left(X_{i}^{L} ; X_{j}^{L}\right)+T_{X_{i} \rightarrow X_{j}},
$$

where $T_{X_{i} \rightarrow X_{j}}$ is the transfer entropy (TE). Probabilities to calculate CaMI are constructed considering the probabilities of the encoded binary symbolic sequences. CaMI is thus calculated by

$$
C_{X_{i} \rightarrow X_{j}}=\sum_{S_{i}, S_{j}} P\left(S_{i}^{L}, S_{j}^{2 L}\right) \log \frac{P\left(S_{i}^{L}, S_{j}^{2 L}\right)}{P\left(S_{i}^{L}\right) P\left(S_{j}^{2 L}\right)},
$$

where the summation indexes $S_{i}$ and $S_{j}$ represent the space of possible length- $L$ symbolic sequences coming from neuron $i$ and $S_{j}$ the space of possible joint events of finding a length- $L$ symbolic sequence coming from neuron $j$ at time $n-L$ followed by a length- $L$ symbolic sequence in this same neuron at time $n$, or in other words, of finding a length- $2 L$ symbolic sequence in neuron $j$ starting at the time $n-L$. $P\left(S_{i}^{L}\right)$ is the probability of finding symbolic sequences $S_{i}^{L}=$ $\left\{s_{i}, \ldots, s_{i}^{L-1}\right\}$ in $X_{i}, P\left(S_{j}^{2 L}\right)$ is the probability of finding a particular length- $L$ symbolic sequence $S_{i}^{2 L}=\left\{s_{i}, \ldots, s_{i}^{2 L-1}\right\}$ in $X_{j}$, and $P\left(S_{i}^{L}, S_{j}^{2 L}\right)$ is the joint probability between length$L$ symbolic sequences in neuron $i$ and length- $2 L$ symbolic sequences in neuron $j$. The directionality index (DI) defined in Ref. [13] in terms of the TE can be calculated by

$$
D_{X_{i} \rightarrow X_{j}}=C_{X_{i} \rightarrow X_{j}}-C_{X_{j} \rightarrow X_{i}} .
$$

For simplicity in notation we consider that

$$
D_{X_{i} \rightarrow X_{j}} \equiv D_{i j}
$$

This index measures the net amount of directed information flowing from $X_{i}$ to $X_{j}$. Thus, if $D_{i j}$ is positive (negative), there is a net amount of information flowing from neuron $i$ to neuron $j$ (from neuron $j$ to neuron $i$ ). Our hypothesis, also sustained by the works of $[17,18]$ and others, is that if there is a directed adjacent connection from neuron $i$ to $j$, thus $D_{i j}$ will be considerably larger than the directionality index of neurons that are not adjacently connected. So, the connection is $X_{i} \rightarrow X_{j}$ if $D_{i j}>h$, the connection is $X_{j} \rightarrow X_{i}$ if $D_{i j}<-h$, and there is no connection if $D_{i j} \cong 0$. In the latter 


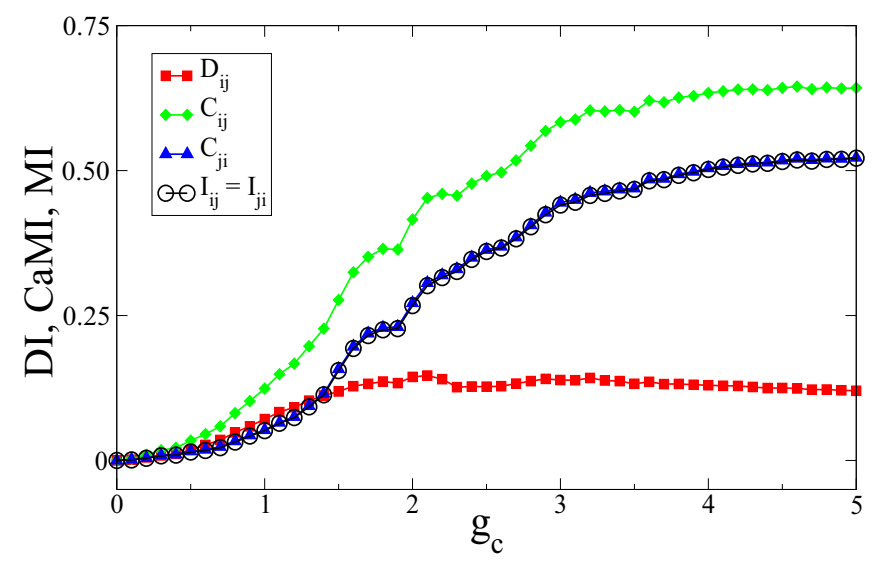

FIG. 2. Directional index (DI), causal mutual information (CaMI), and mutual information (MI), as a function of the coupling strength $\left(g_{c}\right)$ for the mapping step time $\Delta t=0.5 \mathrm{~ms}$ and $L=8$. The red squares, green diamonds, blue triangles, and black circles correspond to $D_{i j}, C_{i j}, C_{j i}$, and $I_{i j}$, respectively.

case, the directionality index will be close to zero because the transfer entropy will be roughly zero for nonadjacent nodes. The mutual information is a symmetric quantity and therefore $I\left(X_{j}, X_{i}\right)=I\left(X_{i}, X_{j}\right)$.

In Fig. 2 we calculate DI $\left(D_{i j}\right)$, CaMI $\left(C_{i j}\right.$ and $\left.C_{j i}\right)$, and MI $\left(I_{i j}\right)$ as a function of $g_{c}$ for two coupled neurons with one directional connection from $x_{i}$ to $x_{j}$. We observe that $D=0$ when the neurons are uncoupled $\left(g_{c}=0\right)$, and $D>0$ for $g_{c}>$ 0 . The information is transmitted from $x_{i}$ to $x_{j}$, in accordance with the direction of the connection. We verify that $C_{j i} \approx I_{i j}$, therefore $T_{j i} \approx 0$ and $T_{i j} \approx D_{i j}$. For the following analysis, we fix $\Delta t=0.25 \mathrm{~ms}$, and $L=8$ that maximizes DI values.

\section{INFERENCE OF TOPOLOGY AND NATURE OF SYNAPSES}

\section{A. Random networks}

We build a directed network where the connections among the neurons are randomly chosen. We consider a random neuronal network with $64 \mathrm{HR}$ neurons and average degree of connectivities $K$ equal to 4 . As a consequence, the network has 256 of a total of 4096 directed connections $(i j)$. Figure 3 shows the normalized directional index, ranked from larger to smaller values, for three different neuronal connectivity configurations: 256 excitatory synapses (black line), 256 inhibitory synapses [red line (dark gray)], and 128 excitatory and 128 inhibitory synapses [green line (light gray)]. In Fig. 3(a) there are two regions with $D_{i j} \neq 0$, that represent the connections from $i$ to $j$, while $D_{i j} \approx 0$ corresponds to the situation in that there is no connection between $i$ and $j$. The magnification [Fig. 3(b)] exhibits two abrupt transitions. The transition to $D_{i j} \approx h$ allows the detection of directed connections in the neuronal network. The transition that occurs for $D_{i j}>h$ allows one to infer the excitatory and inhibitory synapses, as shown by the dashed blue line, where we observe the existence of 128 excitatory and 128 inhibitory synapses.

Notice that the DI values between adjacent and nonadjacent neurons are notably dissimilar, meaning that a small threshold
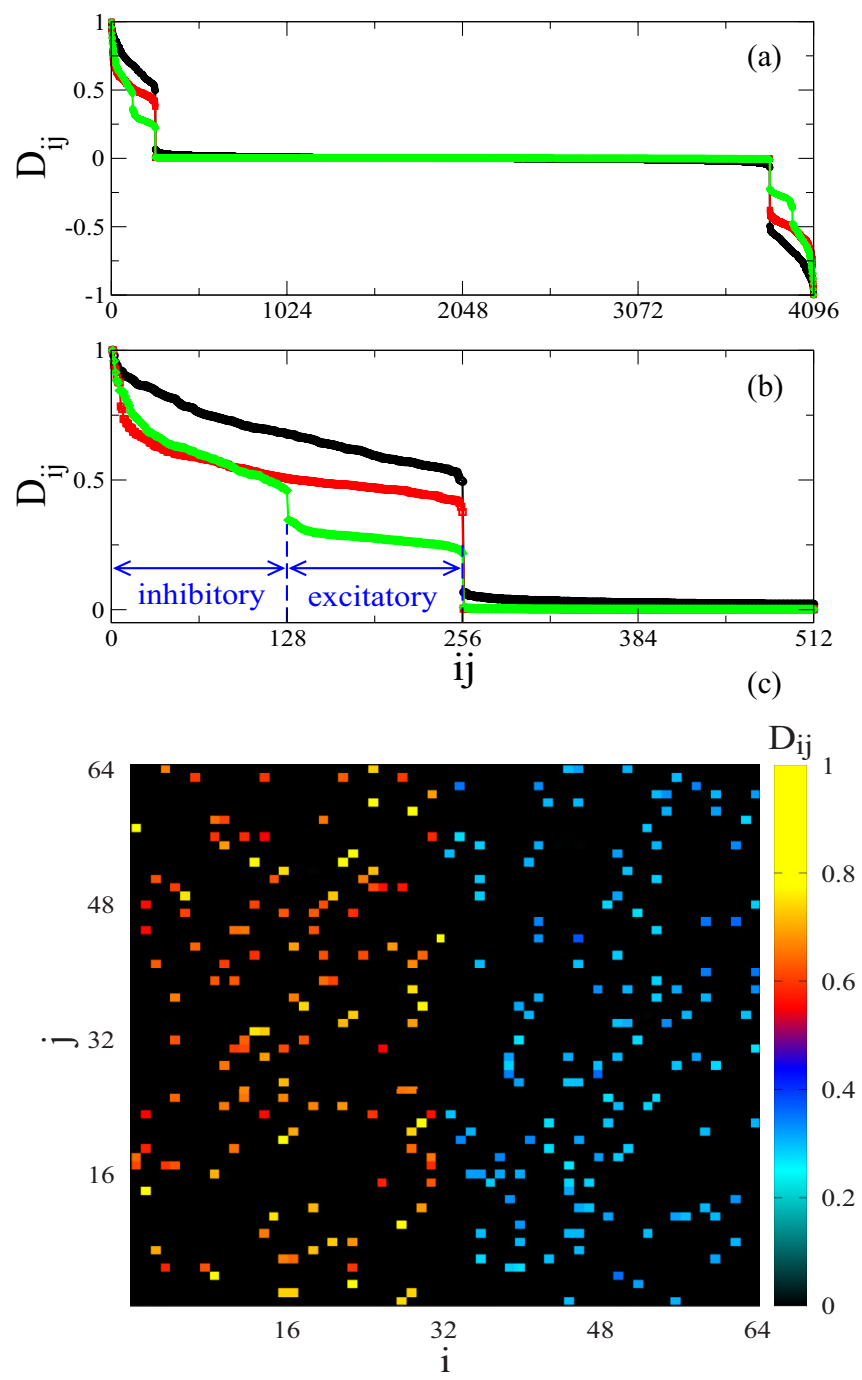

FIG. 3. (a) Normalized directional index, ranked from larger to smaller values, for a random neuronal network with $N=64 \mathrm{HR}$ neurons, $K=4, \Delta t=0.25, L=8, T=410^{6}$, and $g_{c}=0.1$. We consider three cases for the connectivity: 256 excitatory synapses (black line), 256 inhibitory synapses (red line), and 128 excitatory and 128 inhibitory synapses (green line). (b) Magnification of (a). (c) Matrix of the normalized directional index $\left(D_{i j}\right)$ of the latter case.

$h$ can be chosen such that $D_{i j}>h$ implies a directed connection from neuron $i$ to neuron $j$. For the network whose neurons are connected by both inhibitory and excitatory synapses, we notice in the green (light gray) line of Fig. 3 two ranges of DI dissimilar values. For $h<D_{i j}<0.4$, the connection is excitatory and for $D_{i j}>0.4$ the connection is inhibitory. In Fig. 3(c) we see the adjacency matrix, where the colored (gray scale) elements of the matrix indicate if the pairs of neurons are connected. The uncoupled pairs of neurons are indicated in black, while the coupled pairs are in color scale according to the normalized directional index. We consider the same parameters used to calculate the green (light gray) line in Fig. 3. For $D_{i j}<0.4$ the color scale shows the excitatory synapses and for $D_{i j} \geqslant 0.4$ the synapses are inhibitory.

We analyze the noise effect in the inference of the connections. Neuronal noise can be related to several sources, such 


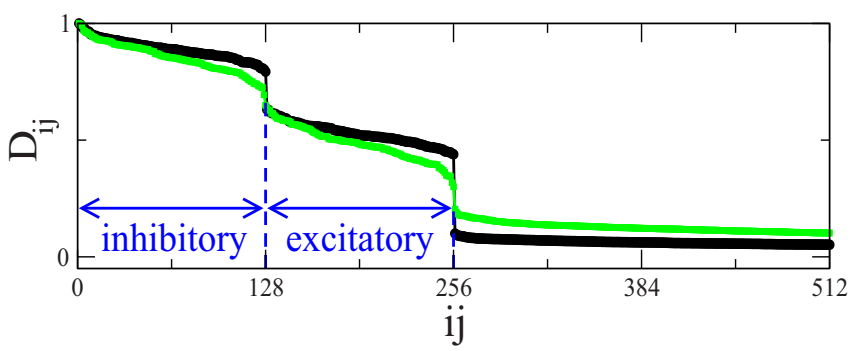

FIG. 4. Normalized directional index for $\sigma_{d}=3$ (black line) and $\sigma_{d}=4$ (green line). We consider $\Delta t=0.25, L=8$, and $g_{c}=0.15$.

as synaptic noise [34] and ion conductance noise [35]. In the action potential equation, we add a Gaussian noise with zero mean and variance $\sigma_{d}$. We calculate the $D_{i j}$ values for the neuronal network with $\sigma_{d}=3$ (black line) and $\sigma_{d}=4$ (green line), as shown in Fig. 4. We verify that the inference for the existence of a synapse is robust to dynamic noise in the membrane potential. However, for $\sigma_{d} \gtrsim 3.5$ it is not possible to infer whether the synapse is excitatory or inhibitory. Therefore, the inference of the connectivities is more robust than the inference of its nature of the synapses.

We also consider an additive noise to analyze the CaMIbased inference. The additive noise is related to the imprecision of the equipment responsible for capturing the electrical signals in the neural membrane, so in our simulations we add to the values of $p(t)$ a noise with zero mean and standard deviation $\sigma_{a}$. In Figs. 5(a) and 5(b) we observe the change in the dynamics of the membrane potential of a network neuron
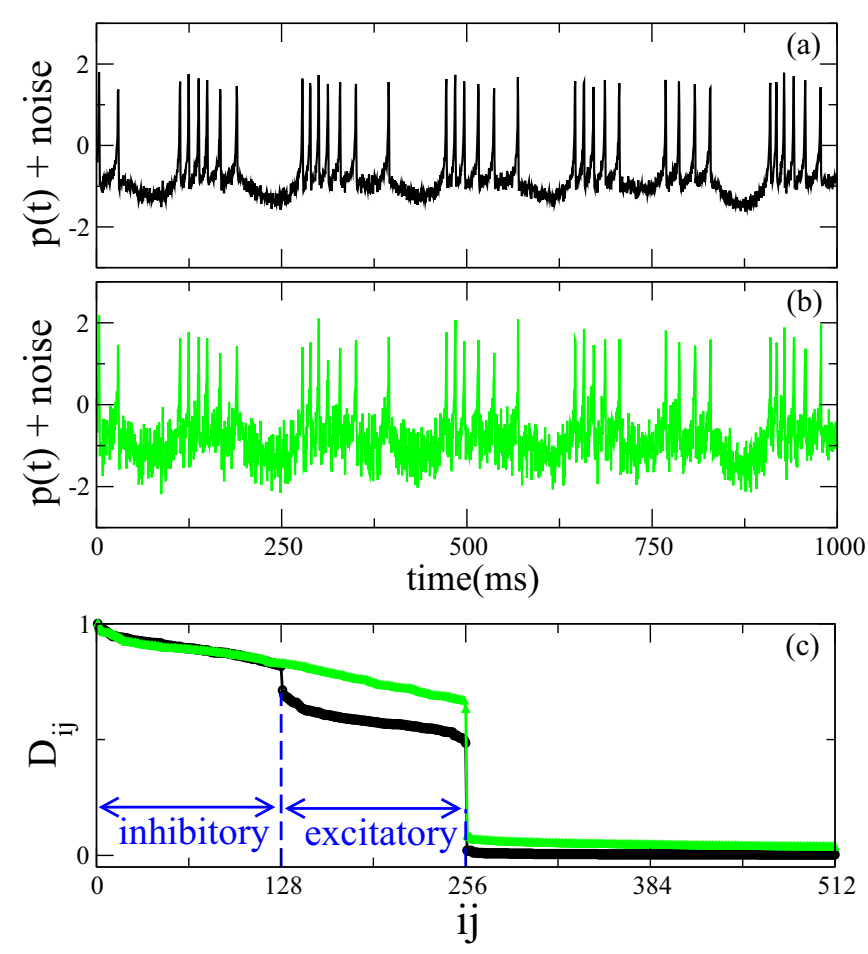

FIG. 5. Membrane potential for the neuron $i=32$ for additive noise standard deviation (a) $\sigma_{a}=0.1$ and (b) $\sigma_{a}=0.35$. (c) Normalized directionality index for $\sigma_{a}=0.1$ (black line) and $\sigma_{a}=0.35$ (green line). We consider $\Delta t=0.5, L=4$, and $g_{c}=0.1$.

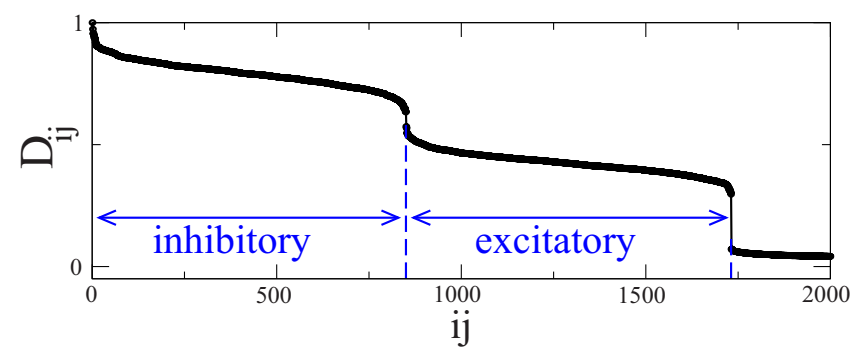

FIG. 6. Normalized directional index, ordered from larger to smaller values for $N=277 \mathrm{HR}$ neurons. We consider $\sigma_{d}=1, g_{c}=$ $0.035, \Delta t=0.25$, and $L=8$.

under the application of additive noise with $\sigma_{a}=0.1$ and $\sigma_{a}=0.35$, respectively. The difference between the minimum and maximum values reached by the membrane potential of the HR model is approximately 3.5 , so $\sigma_{a}=0.35$ corresponds to $10 \%$ of this value. For $\sigma_{a}=0.1$ the observed dynamics remains very similar to the case with no noise observed in Fig. 1, however, when $\sigma_{a}=0.35$ the noise intensity can change the values of the symbolic sequence $S_{i}^{L}(n)$. In Fig. 5(c) we see that the DI calculation does not present significant changes when considering the additive noise with $\sigma_{a}=0.1$ (black line). For $\sigma_{a}=0.35$ (green line) it is no longer possible to distinguish excitatory connections from inhibitory ones, but all 256 connections are detected. Therefore, CaMI-based inference is also robust to additive noise of moderate amplitude.

\section{B. C. elegans neuronal network}

In the literature, there are many works that consider C. elegans neuronal network to study the nervous system $[36,37]$. The C. elegans is a soil worm with body size about $1 \mathrm{~mm}$ and a simple nervous system [38]. We consider in our study the connectome of the large somatic nervous system according to Ref. [39] that consists of 277 neurons. To test our inference approach, we consider approximately 50\% of excitatory and $50 \%$ of inhibitory synapses in the C. elegans network with 1731 directed connections. The directed adjacency matrix $\left(\varepsilon_{i j}\right)$ is obtained from the brain connectivity of the $C$. elegans. Figure 6 exhibits the DI values, where the two discontinuity transitions in the DI values correspond to the excitatory and inhibitory synapses. In Fig. 6 it is possible to identify the connected neurons of the $C$. elegans, where from $i=1$ to $i=138$ and from $i=139$ to $i=277$ there are 850 inhibitory synapses and 881 excitatory synapses, respectively.

\section{INFORMATION FLOW BETWEEN NETWORKS}

In many experimental cases it is not possible to directly measure the membrane potential of each neuron, but only an average field of a group of them, or a brain region. Through the analysis of the mean field between two neural networks, we show that it is possible to infer if distinct networks are connected to each other, and identify the direction of the effective connectivity by the direction of the flow of information.

In order to do this analysis, we considered two random networks with $N=64$ neurons each, with an average degree 
(a)
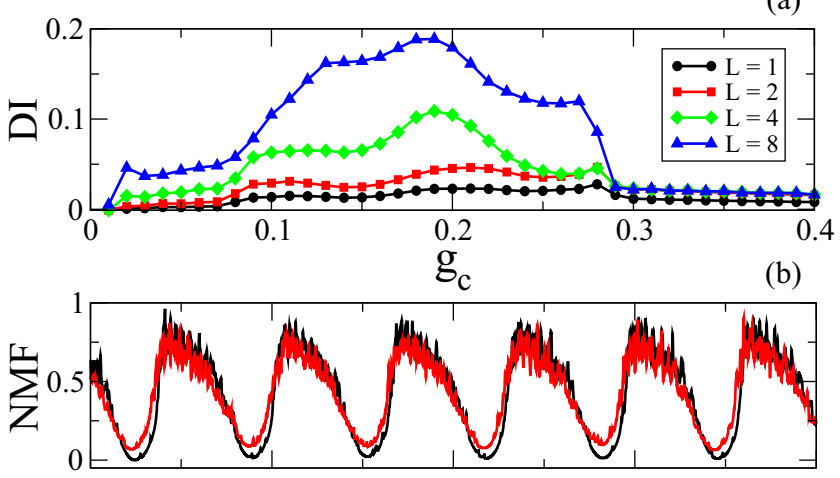

(c)

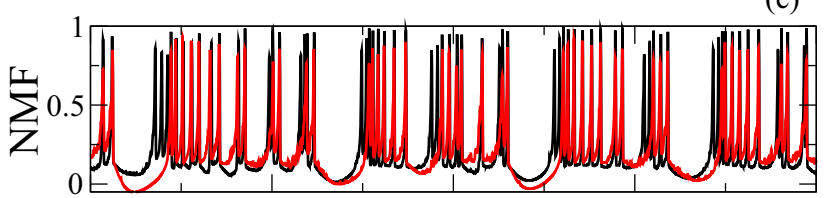

(d)

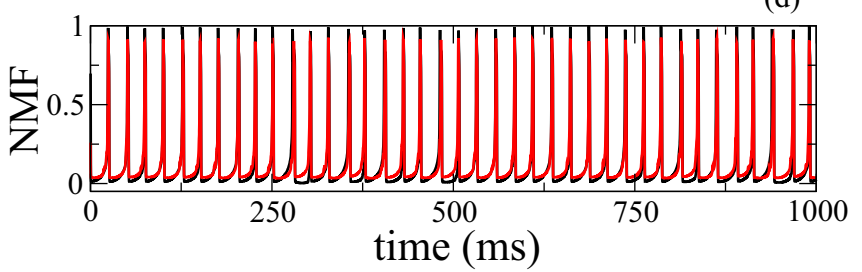

FIG. 7. (a) Directionality index (DI) as a function of coupling strength $\left(g_{c}\right)$. For $\Delta t=0.25 \mathrm{~ms}$, where $L=1$ (black circles), $L=2$ (red squares), $L=4$ (green diamonds), and $L=8$ (blue triangles). Time evolution of the normalized mean field (NMF) for network 1 (black line) and network 2 (red line), both with (b) $g_{c}=0.025$, (c) $g_{c}=0.175$, and (d) $g_{c}=0.275$.

of intraconnections within the networks $K_{\text {intra }}=24$ and an average degree of interconnections between networks $K_{\text {inter }}=$ 12. To study the flow of information between the two networks, we consider that there are only directed connections from neurons of network 1 to neurons of network 2 . In each of the networks we calculated the mean field of the membrane potential and made the symbolic sequence using this time series. The process of calculating DI was performed in the same way as in the case of isolated neurons.

In Fig. 7(a) we show the values obtained from the DI as a function of the intensity of the coupling $g_{c}$, where we set $\Delta t=0.25 \mathrm{~ms}$ and we evaluate different sizes for the symbolic trajectory: $L=1$ (black line), $L=2$ (red line), $L=4$ (green line), and $L=8$ (blue line). We find that, as in the case of two neurons, the highest DI values are observed when using symbolic trajectories of size $L=8$. In this case, we observed that when the coupling is low, the DI values are small, since the influence of network dynamics 1 on network 2 is smaller. For a coupling around $g_{c}=0.175$ we have the highest calculated value of DI and for $g_{c}>0.275$ the value of DI decreases, tending to a constant value. This happens when the neurons of both networks are roughly completely synchronous. The neurons had been completely synchronous, thus the transfer entropy would be zero, resulting in a DI of zero. To understand more about the dynamical behavior leading to the curve presented by the blue line in Fig. 7(a), we analyze the temporal evolution of the normalized mean field (NMF) for three values of the coupling. In Fig. 7(b) we have $g_{c}=0.025$ and we observe that the NMF of network 1 (black line) and network 2 (red line) show that the neurons of these networks present the behavior of bursting synchronization, when neurons start the bursting of firing activities roughly simultaneously. Firings are asynchronous. In Fig. 7(c) we have $g_{c}=0.175$ and the NMF of networks 1 and 2 show that not only intra- but also interneurons are roughly synchronous. Firing spikes in the NMF indicates intrasynchronization. Intersynchronization is evidenced by the fact that the curves are roughly identical. Both of these factors are responsible for the high DI values. Finally, in Fig. 7(d) we have $g_{c}=0.275$ which is intense enough to make the networks almost fully synchronized.

Therefore, even in the case when we have only the data of the average field of networks, we show that it is possible to infer the effective directionality of the connections in a similar way to the case between two neurons only. This method may be thus suitable to be considered for information flow studies in different regions of the brain, analyzing data obtained from several experimental sources such as structural and functional MRI, diffusion tensor imaging, magnetoencephalography, and electroencephalography.

\section{CONCLUSION}

In conclusion, we propose a successful methodology based on CaMI to infer, characterize, and investigate the transmission of information in neuronal networks with chemical synapses. Through the CaMI, we show not only how to infer the existence of synapses, but also to identify the nature of the synapse. Our technique can be applied to the time series generated with Gaussian dynamical noise built-in in the neuron equations, or to the time series contaminated by observational noise. Moreover, we also showed that when access to the neuron potential is not possible, but rather only local mean fields can be measured, such as those coming from EEG signals, our CaMI-based technique can correctly determine the effective net directed connectivity between different neuronal clusters. This work also shows that excitatory connections are not so efficient to transfer information as inhibitory connections, and that nonadjacent neurons transfer roughly zero amount of information. This latter observation suggests that a presynaptic neuron (a neuron that has an adjacent connection to the postsynaptic one) not only exchanges information (positive mutual information), but is also capable of using information to cause an effect in a postsynaptic neuron (positive transfer entropy). Nonadjacent neurons only exchange information. This one-to-one relationship between structure and information transmission remains valid for a wide range of the coupling strength $g_{c}$, constraint within an interval with not so small (to prevent full decorrelation) and not so large (to prevent full synchronization) bounds. For an inference with no mistakes, the time series should be sufficiently long, more specifically, their size in seconds (i.e., $t$ ) should scale with the size of the network, and it is a function of the coupling strength, a relationship that was studied in much detail in Ref. [17]. When dealing with real data, a method for removing bias due 
to limited data points should be applied. Another option would be to do several experiments and do the analysis considering all the samples from all the experiments.

In future works, we plan to consider a model with spike timing-dependent plasticity [40] to study the transfer of information between not only adjacent but also nonadjacent neurons.

\section{ACKNOWLEDGMENTS}

CAPES, DFG-IRTG 1740/2, Fundação Araucária, Newton Fund, CNPq (Grants No. 154705/2016-0 and No. 311467/2014-8), FAPESP (Grants No. 2011/19296-1, No. 2015/07311-7, No. 2016/16148-5, No. 2016/23398-8, and No. 2015/50122-0), and EPSRC Ref. EP/I032606/1.
[1] A.-L. Barabási and Z. N. Oltvai, Nat. Rev. Genet. 5, 101 (2004).

[2] M. P. Niemira and T. L. Saaty, Int. J. Forecasting 20, 573 (2004).

[3] A. Arenas, A. Díaz-Guilera, J. Kurths, Y. Moreno, and C. Zhow, Phys. Rep. 469, 93 (2008).

[4] E. Bullmore and O. Sporns, Nat. Rev. Neurosci. 10, 186 (2009).

[5] G. Gong, Y. He, L. Concha, C. Lebel, D. W. Gross, A. C. Evans, and C. Beaulieu, Cerb. Cortex 19, 524 (2009).

[6] H. X. Ta, C. N. Yoon, L. Holm, and S. K. Han, BMC Syst. Biol. 4, 70 (2010).

[7] A. M. Bastos and J.-M. Schoffelen, Front. Syst. Neurosci. 9, 1 (2016).

[8] M. Takigawa, G. Wang, H. Kawasaki, and H. Fukuzako, Int. J. Psychophysiol. 21, 65 (1996).

[9] A. Baccalá and K. Sameshima, Biol. Cybern. 84, 463 (2001).

[10] X.-M. Cui, W. S. Kim, D.-U. Hwang, and S. K. Han, Europhys. Lett. 110, 38001 (2015); Y. Chen, G. Rangarajan, J. Feng, and M. Ding, Phys. Lett. A 324, 26 (2004); N. Ancona, D. Marinazzo, and S. Stramaglia, Phys. Rev. E 70, 056221 (2004).

[11] M. P. van den Heuvel and H. E. H. Pol, Eur. Neuropsychopharmacol. 20, 519 (2010).

[12] P.-O. Amblard and O. J. J. Michel, J. Comput. Neurosci. 30, 7 (2011).

[13] T. Schreiber, Phys. Rev. Lett. 85, 461 (2000).

[14] J. Runge, Phys. Rev. E 92,062829 (2015).

[15] J. Sun, D. Taylor, and E. M. Bolt, SIAM J. Appl. Dyn. Syst. 14, 73 (2015).

[16] W.-X. Wang, Y.-C. Lai, and C. Grebogi, Phys. Rep. 644, 1 (2016).

[17] N. Rubido, A. C. Martí, E. Bianco-Martínez, C. Grebogi, M. S. Baptista, and C. Masoller, New J. Phys. 16, 093010 (2014).

[18] E. Bianco-Martinez, N. Rubido, C. G. Antonopoulos, and M. S. Baptista, Chaos 26, 043102 (2016).
[19] J. Massey, in Proceedings of the International Symposium on Information Theory and Its Applications, Waikiki, Hawai, USA (IEEE, Piscataway, 1990), pp. 27-30.

[20] I. Vlachos and D. Kugiumtzis, Phys. Rev. E 82, 016207 (2010).

[21] J. L. Hindmarsh and R. M. Rose, Proc. R. Soc. London B 221, 87 (1984).

[22] A. Pereda, Nat. Rev. Neurosci. 15, 250 (2014).

[23] Y. Liu and S. Aviyente, Comp. Math. Methods Med. 2012, 635103 (2012).

[24] B. Schelter, M. Mader, W. Mader, L. Sommerlade, B. Platt, Y.-C. Lai, C. Grebogi, and M. Thiel, Europhys. Lett. 105, 30004 (2014).

[25] E. J. Bianco-Martinez, Ph.D thesis, University of Aberdeen, 2015.

[26] E. Bianco-Martinez and M. S. Baptista, arXiv:1612.05023v1.

[27] F. Montani, E. B. Deleglise, and O. A. Rosso, Physica A 401, 58 (2014).

[28] A.-L. Barabási and R. Albert, Science 286, 509 (1999).

[29] S. Timotheou, Comput. J. 53, 251 (2010).

[30] E. Gelenbe, Neural Comput. 1, 502 (1989).

[31] M. Kaiser and C. C. Hilgetag, PLoS Comput. Biol. 2, e95 (2006).

[32] J. G. White, E. Southgate, J. N. Thomson, and S. Brenner, Phil. Trans. R. Soc. Lond. B 314, 1 (1986).

[33] A. L. Hodgkin and A. F. Huxley, J. Physiol. 117, 500 (1952).

[34] G. Györgyi, Phys. Rev. Lett. 64, 2957 (1990).

[35] X. J. Cao and D. Oertel, J. Neurophysiol. 94, 821 (2005).

[36] K. S. Liu and P. W. Sternberg, Neuron 14, 79 (1995).

[37] L. R. Varshney, B. L. Chen, E. Paniagua, D. H. Hall, and D. B. Chklovskii, PLoS Comput. Biol. 7, e1001066 (2011).

[38] C. Gally and J. L. Bessereau, Med Sci (Paris) 19, 725 (2003).

[39] Connectome File Format Datasets (Version 2.0) [http://cmtk.org/viewer/datasets/].

[40] J.-P. Pfister and W. Gerstner, J. Neurosci. 26, 9673 (2006). 